\title{
Optical Character Recognition using Global and Local Features with Modified K-means and Neural Network
}

\author{
Chin-Sheng Chen ${ }^{\mathrm{a}, *}$, Wen-Chun Yang ${ }^{\mathrm{a}}$, Chien-Liang Huang ${ }^{\mathrm{a}}$ \\ ${ }^{a}$ Institute of Automation Technology, National Taipei University of Technology, 1, Sec. 3, Zhongxiao E. Rd., Taipei \\ 10608, Taiwan (R.O.C) \\ *Corresponding Author: saint@mail.ntut.edu.tw
}

\begin{abstract}
This paper presents a hierarchical classification of Optical Character Recognition (OCR) using global and local features with modified k-means and neural network. A hierarchical classification strategy, which contains training and recognition phases, was designed to precisely recognize the characters.

In the training phase, the global features of sample characters and modified k-means clustering are employed to quickly categorize the characters into several clusters. Then each cluster and its corresponding local features of sample characters are fed into the Back-Propagation Neural Network (BPN) to learn the optimal weights. In the recognition phase, both global and local features are extracted from the test characters. Then the character is recognized by well-trained clusters' center and neural network for coarse and fine recognition, respectively. Experimental results of the three different datasets tested showed classification rates of $97.22 \%, 100 \%$ and $90.28 \%$, respectively.
\end{abstract}

Keywords: optical character recognition, global and local features, hierarchical classification, back-propagation neural network.

\section{Introduction}

Optical character recognition (OCR) system is widely applied in industrial applications ${ }^{(1)}$, and it has become one of the most essential applications of technology in the field of pattern recognition and artificial intelligence. It contributes significantly to the advancement of automation process and improves the interface between human and machine. In general, character recognition algorithm consists of two main stages: feature extraction and classification.

Both feature extraction and selection are very important in achieving high recognition performance of OCR system. Several feature extraction techniques for character recognition have been reported ${ }^{(2,3)}$. The identifiable features extracted from the segmented characters are improving the recognition rate and reducing misclassification. Besides feature extraction, feature selection also has tremendous effects on the identification result; therefore, the selected features must be sufficiently discriminable to each segmented character and powerful enough to classify all relevant classes correctly. In addition, the classification technique is also of vital importance in the OCR system. Many classification techniques have been introduced $^{(4)}$, including statistical methods, Artificial Neural Networks (ANNs), Support Vector Machines (SVM) and multiple classifier combination. The strengths and weaknesses of each classification technique have also been discussed. Among several methods proposed, the artificial neural network has been most frequently used as a powerful tool for classification problems.

Sophisticated neural network classifiers and novel feature extraction techniques have been proposed for achieving high recognition performance ${ }^{(5-8)} . \mathrm{In}^{(5)}$, different classifiers, including multiple Multilayer Perceptron (MLP), Hidden Markov Model (HMM) and structure adaptive self-organizing map (SOM) are employed to solve difficult problems such as handwritten numeral recognition. The results show that the multiple MLP classifier is more preferable than the others. For recognition accuracy, novel feature extraction techniques have been proposed ${ }^{(6,7)}$. In this study, features are obtained from three different orientations, horizontal, vertical and diagonal directions, and they obviously increase the recognition rate for handwritten character recognition system. Hybrid features are 
introduced to recognize English handwritten characters in ${ }^{(8)}$, and the hybrid features are obtained by combining the features extracted using diagonal, directional, Principal Component Analysis (PCA) and geometry feature extraction techniques. The hybrid features thus obtained are fed into the feed forward propagation neural network for classification tasks. In contrast, other methods involving different classification strategies are proposed. An excellent hierarchical classification was performed $\mathrm{in}^{(9)}$. In this approach, the features are extracted at different resolution levels and those features consider only the sub region in the image that meets certain acceptance criteria at higher level. In $^{(10,11)}$, another hierarchical classification approach involving new structural features was introduced, in which features are extracted using recursive subdivisions of the character image in a hierarchical approach. Lower levels are utilized to perform preliminary discrimination whereas higher levels help distinguish between characters of similar shapes that are confusing when using only lower levels. As mentioned above, various features are computed and extracted in the OCR system, although it is possible that the same extracted features do not have any efficacy in the recognition stage. Therefore, redundant feature may degrade the recognition results, and increase the time complexity of the recognition process ${ }^{(12)}$.

This paper proposes a hierarchical classification approach of OCR using global and local features with modified k-means and neural network. This approach combines four different features, namely, aspect ratio, skeleton area, geometrical distance and diagonal feature. The two-stage study is implemented using a coarse-to-fine scheme where characters are classified into a possible group in the first stage and the test characters are then correctly recognized in the second stage. Although the proposed approach regards only a few features as inputs of the neural network, high recognition accuracy is achieved.

The remaining sections of this paper are organized as follows. In Section 2, the proposed hierarchical OCR algorithm is presented. Section 3 describes the feature extraction methodology. Section 4 presents classification methods while experimental results are discussed in Section 5. Finally, conclusions are drawn in Section 6.

\section{Proposed hierarchical OCR algorithm}

The main procedure of the proposed algorithm is shown in Fig. 1. As can be seen, It consists of two phases: training phase and recognition phase. In the training phase, the global and local features are extracted from the sample characters. Then the global features are fed into the modified k-means clustering. After the clustering method, the sample characters are grouped into a few clusters, $k$. Furthermore, the center of each cluster, $C_{k}$, is also recorded. Next, the weights of BPN for each cluster, $W_{k L}$, are trained using local features.

Then $C_{k}$ and $W_{k L}$ are fed into the recognition phase to recognize the test characters. In the recognition phase, global features are extracted from the test characters, thus dividing the characters into specific clusters $G_{k}$ according to the distance between the global features of the test character and each center of the training features in the first stage. In the second stage, both local features and well-trained weights $W_{k L}$ are employed to correctly

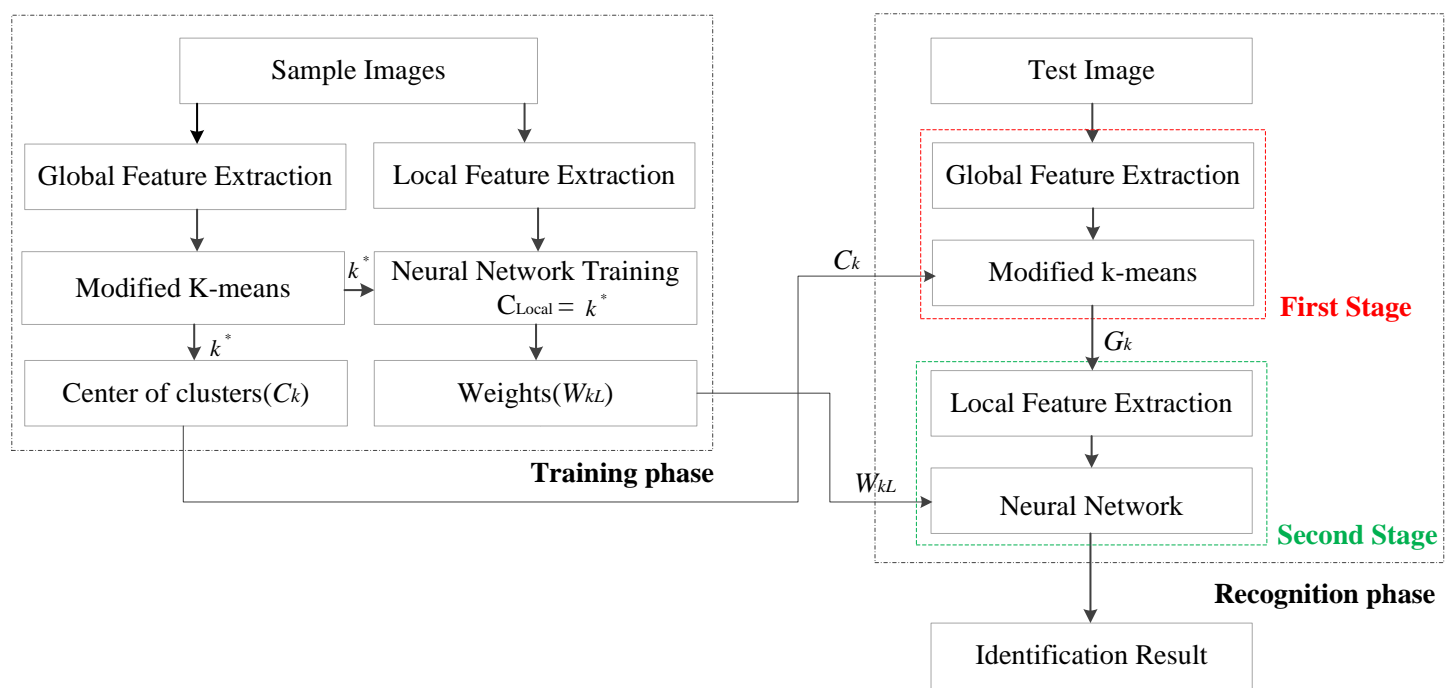

Fig. 1. Architecture of the proposed algorithm. 
recognize the test characters. Moreover, the proposed two-stage strategy increases the recognition rate when the test characters have changes in thickness and deformation.

\section{Feature extraction}

The most critical aspect of character recognition is how to select suitable features for feature extraction. The selected features should be distinct and reasonably invariant with respect to variations in character shape caused by the imaging environment. This study used four different approaches for extracting global and local features. In the recognition phase, global features are employed to achieve the preliminary discrimination in first stage of the proposed classification strategy. Relatively, local features are employed to precisely recognize the test characters.

\subsection{Global feature}

The global features are extracted using three methods, namely aspect ratio, skeleton area and geometrical distance, as shown in Fig. 2 and described below.

Aspect Ratio: The aspect ratio describes the proportional relationship between the width and height of bounding rectangle of the character, which is invariant for characters of any size. The feature is computed as

$$
F_{A R}=\frac{\text { Width }}{\text { Height }}
$$

Skeleton Area: Skeletons are important shape descriptors in object representation and recognition. Typically, the skeletons of volumetric models are computed using iterative thinning. Fig. 2(b) shows the skeleton of a character, whose area is summarized in the pixel of skeleton.

Geometrical Distance: This property is a certain particular average distance of the image pixels at the bounding rectangle corners. Here, there are four corner points $\left(P_{1}-P_{4}\right)$ of the bounding rectangle, as seen in Fig. 2(c). The feature

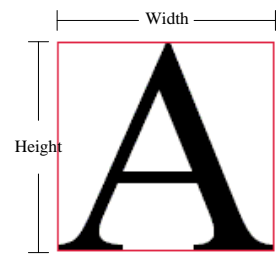

(a) Aspect ratio.

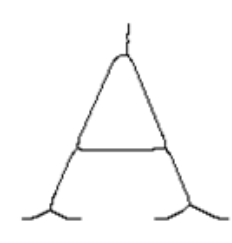

(b) Skeleton

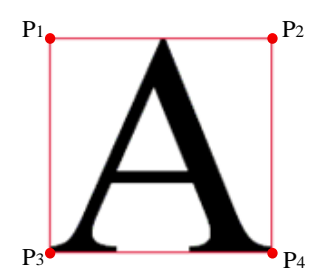

(c) Geometrical distance.
Fig. 2. Global features of the character.

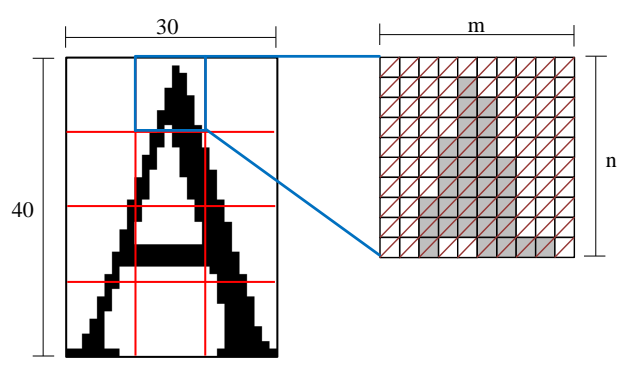

Fig. 3. Local features of the character.

formula is defined in Eq. (2).

$$
\begin{gathered}
F_{G D}=\frac{\sum_{j=1}^{4} \sum_{i=1}^{n} S_{i j}}{4 \cdot n}, j=1,2,3,4 \\
S_{i j}=\sqrt{\left(x_{i}-x_{j}\right)^{2}+\left(y_{i}-y_{j}\right)^{2}}
\end{gathered}
$$

where $n$ is the number of points labeled with object and $S_{i j}$ refers to the distance between the point $i$ located in the object and the corner point $j$.

\subsection{Local feature}

The character image is resized to $30 \times 40$ pixels and the image is divided into $N_{L}$ rectangular sub-images, as shown in Fig. 3. The size of each sub-images is $m \times n$ pixels and it has $(m+n)-1$ diagonal lines in each sub-image. The sub-feature of each diagonal line $D(i)$ is obtained from the sum of all pixels along the corresponding diagonal line. Thus, $(m+n)-1$ sub-features are obtained from each sub-image, then sub-feature values are averaged to obtain a single feature $F_{\text {diagonal }}(r)$ for a sub-image, as

$$
F_{\text {diagonal }}(r)=\frac{\sum_{i=0}^{(m+n)-1} D(i)}{(m+n)-1}
$$

This step is sequentially repeated for all the sub-images, $r$ is from 1 to $N_{L}$. There could be some sub-images whose diagonals are empty of foreground pixels, then the feature values corresponding to these sub-images are zero. Finally, $N_{L}$ features are extracted from each character. In this paper, the value of $N_{L}$ is 12 and both $m$ and $n$ are 10 .

\section{Classification}

For the recognition phase, a hierarchical classification scheme is employed. Characters with similar shapes (i.e., ' 0 ' and ' $\mathrm{O}$ ' and ' 2 ' and ' $\mathrm{Z}$ ', ) often causes confusion, and the proposed two-stage classification is designed to solve this problem. In the first stage, the modified k-means clustering 
tends to quickly find the cluster of comparable extent. The global features are extracted from different sample characters; and the neural network is employed to recognize specific character in each cluster in the second stage.

\subsection{Modified k-means clustering}

Cluster analysis is a useful technique of classifying large amount of data into subsets. Enormous data are thereby organized into an efficient and useful representation that characterizes the cluster being sampled. K-means is one of the most popular and efficient clustering methods that could minimize clustering error. Classic k-means starts with the initial number of cluster and the cluster centers; hence, the performance depends heavily on the initial conditions. To overcome these problems, the max-min principle is proposed to determine the initial conditions [13]. In order to determine the number of clusters to be grouped, the maximum variation of standard deviation between a range of clusters is conducted to obtain the optimal number of clusters [14]. In this paper, the implementation procedure of the modified k-means clustering is shown in Fig. 4. Here, the modified k-means clustering takes the advantages of those literatures, and it can provide more robust clustering result. In the modified k-means clustering procedure, the initial and maximum numbers of cluster are 2 and 9, respectively. Here, the classification performance will

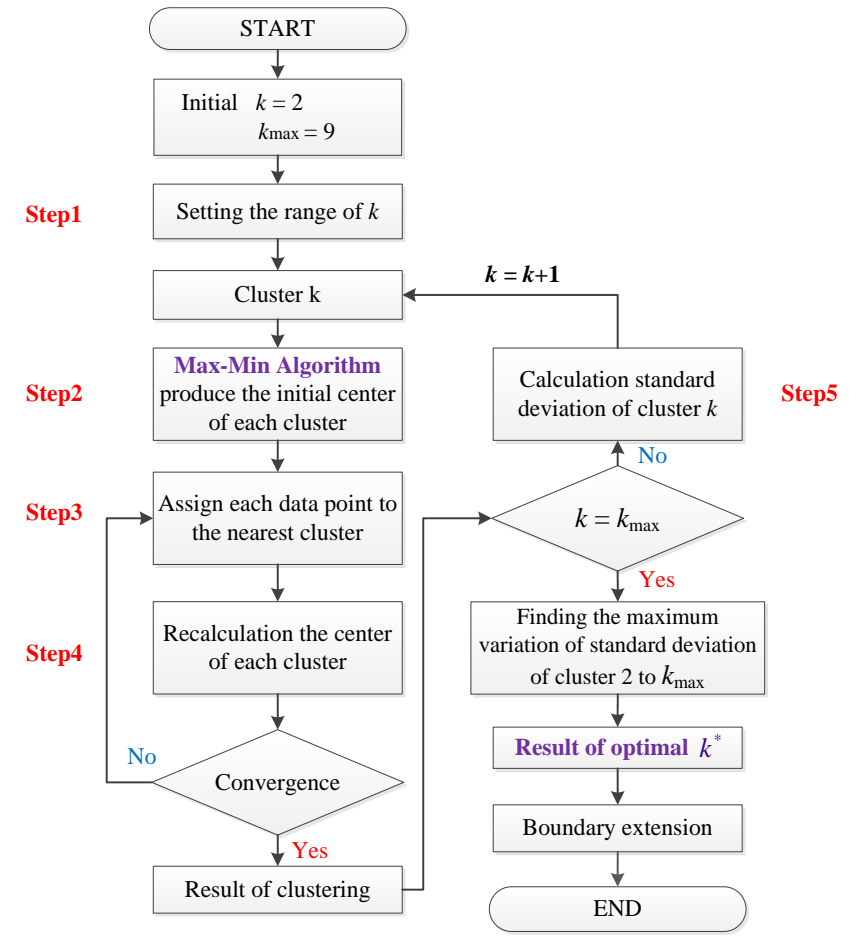

Fig. 4. Procedure of the modified k-means clustering.

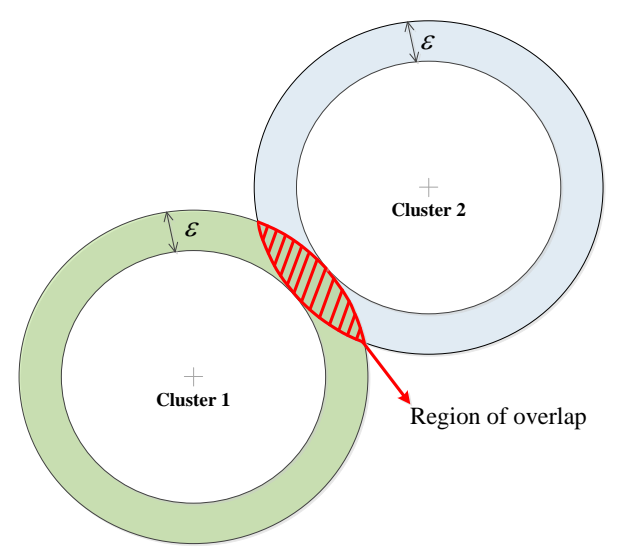

Fig. 5. Boundary layer of cluster.

deteriorate if the maximum number of clusters is less than 2 or more than 9 .

With the optimal clustering result obtained, the boundary layer of each cluster will be extended. This process is very vital to the correct recognition of test characters. After the clustering method, the original boundaries of each cluster are obtained. However, misclassification will occur when the global features of test characters are near the decision boundary in the recognition phase. Therefore, the original boundary is further spread by parameter $\varepsilon$, as shown in Fig. 5, and the clusters are re-defined by the extension boundaries. Hence, if the data are located in the overlapping region, it will belong to both neighboring clusters simultaneously.

There are three kinds of features according to the structural feature calculated by global feature extraction (mentioned in section 3.1), the three-dimensional information is fed into modified $\mathrm{k}$-means clustering to perform the coarse classification in the first stage. The details are illustrated as follows.

Step1: Set a range of $k$. In this study, $k$ is set to range from 2 to 9 .

Step2: Obtain the initial center of each cluster using Max-Min clustering algorithm. This method adopts the farthest distance of each data point to get the center of $k$ cluster.

Step3: Decide which cluster each data point belongs to according to the minimum distance between data point and cluster center.

Step4: Recalculate the center of each cluster using an average of data points in the cluster. Repeat this step until the center values become constant.

Step5: Compute the standard deviation from each $k$ cluster that would be recorded. The standard deviation $S_{k}$ formula is 


$$
S_{k}=\sum_{i=1}^{k} \sum_{j=1}^{n}\left(\frac{\left\|x_{j}-v_{i}\right\|^{2}}{n_{i}}\right)^{\frac{1}{2}}
$$

where $S_{k}$ is the standard deviation of $k$ cluster,$k$ represents the number of clusters, $x$ is the feature point, $v$ is the cluster center, $n$ is the number of feature points.

The standard deviation between $k$ and $l$ cluster $S_{k l}$ is calculated using Eq. (6), and $l$ is $k+1$.

$$
S_{k l}=S_{k}-S_{l}
$$

The optimal number of clusters is selected by $l$ using the maximum of $S_{k l}$ as in Eq. (7).

$$
\arg _{l} \max \left\{S_{k l}, l=k+1, k=2,3, \ldots, k_{\max }\right\}
$$

\subsection{Neural network (NN)}

In this paper, the Multilayer Perceptron (MLP) of the neural network model is adopted. MLP is known as a supervised network because it requires a desired output to learn the optimal weights. A two-layered neural network with a single hidden layer is shown in Fig. 6. The network is fully connected between adjacent layers. The operation of this network can be regarded as a nonlinear decision-making process. The error back-propagation neural network (BPN) is a common method for training the weights between each layer. In this study, the gradient descent of BPN method is employed to determine the optimal weights.

Given a known input $x=\left\{x_{1}, x_{2}, \ldots, x_{N}\right\}$ and the weight set, each output node yields the output $y_{j}$ of belonging to this class as

$$
y_{j}=f\left\{\sum_{k} w_{j k}^{o h} f\left(\sum_{i} w_{k i}^{h i} x_{i}\right)\right\}
$$

where $w_{k i}^{h i}$ is a weight between the $i^{\text {th }}$ input node and the $k^{\text {th }}$ hidden node, $w_{j k}^{\text {oh }}$ is a weight from the $k^{\text {th }}$ hidden node to the $j^{\text {th }}$ class output, $f$ is an activation function.

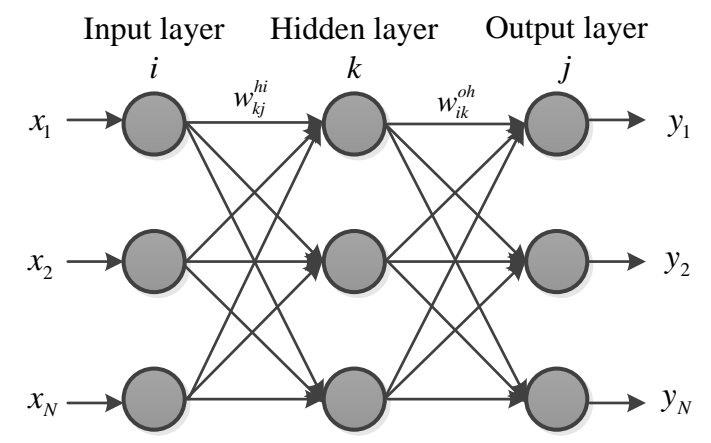

Fig. 6. A two-layered neural network architecture.

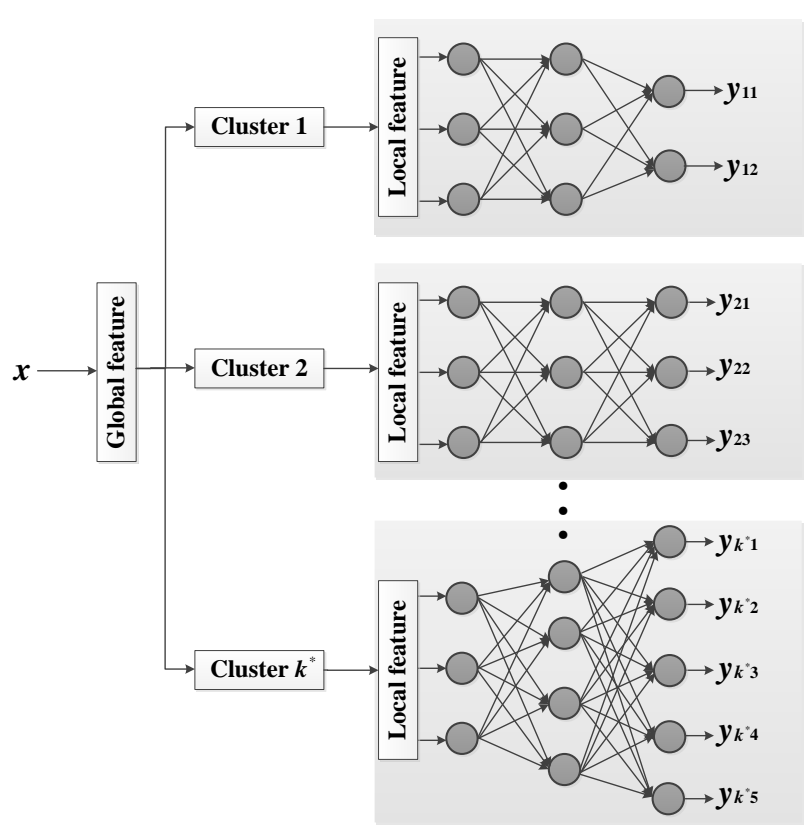

Fig. 7. Architecture of fine classification with neural network.

However, the results are passed through a nonlinear activation function, for the hidden and output units of the activation function are the sigmoid function in this study as shown in Eq. (9). The node having the maximum value is selected as the corresponding class.

$$
f(x)=\frac{1}{1+e^{-x}}
$$

In general, the number of available features and the number of classes are both large in the OCR system. However, a network of a finite size does not often load a particular mapping completely. Therefore, the specific part of complete mapping is considered for a single network, and it would perform its job better. A hybrid method, coarse-to-fine recognition, that considers both global and local features is developed. The modified k-means clustering is a coarse classification for categorizing the test characters into specific networks in the first stage, and the output of the specific network further classifies to correct recognition. The network architecture is shown in Fig. 7.

\section{Experimental results}

Two fonts, Times New Roman and Arial, were tested. The neural network was trained by using three datasets of 26 letters and 10 numbers (108 samples) for each font. In the recognition experiment, the test data make up four datasets (144 samples) that are generated by the morphology technique for each font. The three test 

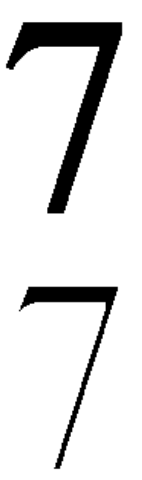

(a) Times New Roman.

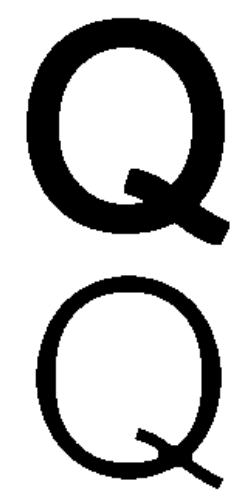

(b) Arial.

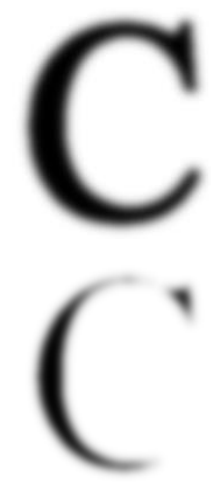

(c) Times New Roman with Gaussian Blur.
Fig. 8. Test characters.

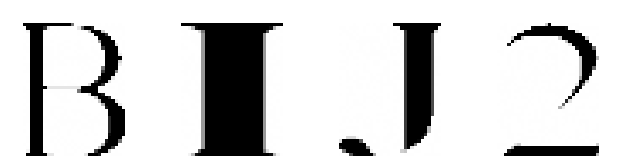

Fig. 9. Misclassification of Times New Roman.

characters of the test datasets are shown in Fig. 8. The experiments were all performed in Visual studio 2010 on a personal computer (PC) with an Intel Core i5 3.1 GHz CPU and $4 \mathrm{~GB}$ of memory. The results of modified k-means is shown in Table 1, the optimal number of cluster is 3 for Times New Roman and 6 for Arial. Table 2 shows the details of the parameters of the neural network, and the parameters are fixed to test. The recognition rates obtained for the proposed classification are shown in Table 3. The cluster boundary layers are $0.25,0.1$ and 0.25 for Times New Roman, Arial cases and Times New Roman with Gaussian blur, respectively. As seen in Table 3, the proposed method has $100 \%$ recognition rate for Arial characters, and $97.22 \%$ recognition rate for Times New Roman characters and $90.28 \%$ recognition rate for Times New Roman with Gaussian blur characters. The misclassification characters for Times New Roman, shown in Fig. 9, are recognized as ' $\mathrm{H}$ ', ' 1 ', ' 3 ' and ' 1 ', respectively. The other misclassification characters for Times New

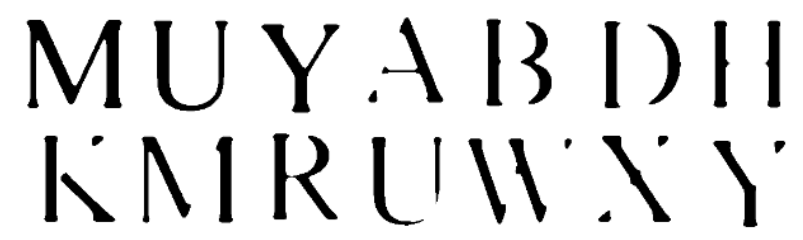

Fig. 10. Misclassification of Times New Roman with Gaussian Blur.
Table 1. Results of the modified k-means.

\begin{tabular}{|c|c|}
\hline Font of character & Optimal number of $k^{*}$ \\
\hline Times New Roman & 3 \\
\hline Arial & 6 \\
\hline
\end{tabular}

Table 2. Details of the proposed neural network.

\begin{tabular}{|l|l|}
\hline Input nodes & 12 \\
\hline Hidden layers & 1 \\
\hline Output nodes & Number of $k$ clusters member \\
\hline Training epochs & 10000 \\
\hline Training algorithm & Gradient descent method \\
\hline Performance function & Mean Square Error (MSE) \\
\hline
\end{tabular}

Table 3. Performance of the proposed classification.

\begin{tabular}{|c|c|c|}
\hline Font of character & $\begin{array}{c}\text { Parameter of } \\
\text { extension } \varepsilon\end{array}$ & Recognition rate \\
\hline Times New Roman & 0.25 & $97.22 \%$ \\
\hline Arial & 0.1 & $100 \%$ \\
\hline $\begin{array}{c}\text { Times New Roman with } \\
\text { Gaussian Blur }\end{array}$ & 0.25 & $90.28 \%$ \\
\hline
\end{tabular}

Roman with Gaussian Blur are shown in Fig. 10. As can be seen, the test characters are incomplete and distorted, thus affecting significantly extraction of both global and local features.

\section{Conclusions}

In this paper, a hierarchical classification strategy using global and local features with the modified k-means and neural network was designed to precisely recognize characters. In the training phase, the number of clusters and the optimal weights of BPN are determined by the global and local features, respectively. In the recognition phase, the coarse-to-fine classification stage is employed to enhance the performance and the recognition rate of test characters with changes in thickness and deformation. The experimental result shows that the proposed method yields excellent recognition accuracy of $97.22 \%, 100 \%$ and $90.28 \%$ for Times New Roman, Arial and blur character, respectively.

\section{References}

(1) Amarjot Singh, Ketan Bacchuwar and Akshay Bhasin : "A survey of OCR applications", 
International Journal of Machine Learning and Computing, Vol. 2, No. 3, pp. 314-318, 2012.

(2) Anil K.Jain and Torfinn Taxt : "Feature extraction methods for character recognition-a survey", Pattern Recognition, Vol. 29, No. 4, pp. 641-662, 1996.

(3) Nafiz Arica and Fatos T. Yarman-Vural : "An Overview of Character Recognition Focused on Off-Line Handwriting", IEEE Transactions on Systems, Man, and Cybernetics, Vol. 31, No. 2, pp. 216-233, 2001.

(4) Cheng-Lin Lin and Hiromichi Fujisawa : "Classification and Learning for Character Recognition: Comparison of Methods and Remaining Problems", Int. Workshop on Neural Networks and Learning in Document Analysis and Recognition, 2005.

(5) Sung-bae Cho : "Neural-network classifiers for recognizing totally unconstrained handwritten numerals", IEEE Transactions on Neural Networks, Vol. 8, No. 1, pp. 43-53, 1997.

(6) J. Pradeep, E. Srinivasan and S. Himavathi : "Diagonal feature extraction based handwritten character system using neural network", International Journal of Computer Applications, Vol. 8, No. 9, pp. 17-22, 2010.

(7) J. Pradeep, E. Srinivasan and S. Himavathi : "Diagonal feature extraction based handwritten alphabets recognition system using neural network", International Journal of Computer Science \& Information Technology, Vol. 3, No. 1, pp. 27-38, 2011.

(8) J. Pradeep, E. Srinivasan and S. Himavathi : "An investigation on the performance of hybrid features for feed forward neural network based English handwritten character recognition system", Wseas Transaction on Signal Processing, Vol. 10, pp. 21-29, 2014.

(9) Jaehwa Park, Venu Govindaraju and Sargur N. Srihari : "OCR in a hierarchical feature space", IEEE Transactions on Pattern Analysis and Machine Intelligence, Vol. 22, No. 4, pp. 400-407, 2000.

(10) G. Vamvakas, B. Gatos and S.J. Perantonis : "Hierarchical classification of handwritten characters based on novel structural features", Eleventh International Conference on Frontiers in Handwriting Recognition, 2008.

(11) G. Vamvakas, B. Gatos and S.J. Perantonis : "Handwritten character recognition through two-stage foreground sub-sampling", Patten Recognition, pp. 2807-2816, 2010.

(12) Mohammad Amin Shayegan and Saeed Aghabozorgi : "A new dataset size reduction approach for PCAbased classification in OCR application", Hindawi Publishing Corporation Mathematical Problems in Engineering, 2014.

(13) Cheng Wang, Junqing Bai, Jiaojiao Li, Longlei Dong, Guirong Yan and Zengguo Sun : "Max-min K-means clustering algorithm and application in response signal feature extraction", International Journal of Applied Electromagnetic and Mechanics, pp. 719-724, 2012.

(14) Ya-Chu Chang : A modified k-means algorithm for pattern identification on monitoring care systems, Master dissertation of National Taipei University of Technology, 2011. 